\title{
因HAD
}

ISSN-L: 2530-5115

DOI: http://doi.org/10.22585/hospdomic.v4i4.117

\section{Utilidad de los relojes inteligentes en las unidades de hospitalización a domicilio}

Usefulness of smartwatches in hospital at home units

Antonio Martin-Marco', Jorge Santander-López ${ }^{2}$

1. Hospital Universitario de La Ribera, Alzira, España

2. Hospital Universitario de La Ribera, Alzira, España

Correspondencia/Correspondence

Antonio Martin-Marco

martin_antmar $1 @$ gra.es

Recibido/Received

06.08.2020

Aceptado/Accepted

01.10 .2020
Conflicto de Intereses/Competing interest

Sin financiación.

Agradecimientos/Acknowledgments

Los autores declaran la inexistencia de conflicto de interés. 


\section{CARTA A LA DIRECTORA}

\section{Sra. Directora.}

La fibrilación auricular es la arritmia cardiaca mantenida más frecuente. Su frecuencia estimada a nivel mundial se sitúa en torno a 33 millones de personas y continúa en aumento. Esto conlleva un exceso de mortalidad, debido fundamentalmente a ictus e insuficiencia cardiaca (1).

En los últimos años han aparecido nuevos dispositivos que permiten la monitorización de las constantes vitales de manera continua, entre ellos destacan los conocidos como relojes inteligentes (smartwatches), que en algunos casos permiten, incluso la realización de electrocardiogramas (ECG) en tiempo real. Existe ya bastante evidencia en la literatura de la utilidad de estos dispositivos a la hora de detectar arritmias cardiacas en las personas que los llevan. Sin embargo, no hemos encontrado publicaciones en las que se hayan utilizado los dispositivos del personal médico, para realizar electrocardiogramas en pacientes de manera que se pueda realizar una primera aproximación diagnóstica, de forma rápida y fiable a la cabecera del paciente (2).

Algunos estudios se han centrado en la realización de ECG con varias derivaciones, permitiendo incluso la detección de trazados que permiten el diagnóstico del infarto agudo de miocardio (IAM), e incluso el alargamiento del segmento QT (medida del tiempo entre el comienzo de la onda Q y el final de la onda T en el electrocardiograma), lo que puede ser prometedor, sobre todo de cara a la pandemia por COVID-19 ya que el tratamiento con antipalúdicos y/o azitromicina produce alargamiento del QT y su determinación mediante smartwatches puede facilitar una detección precoz, sin necesidad de trasladar al paciente de su domicilio $(3,4)$.

Dentro del ámbito de la hospitalización domiciliaria, existen dispositivos portátiles para la realización de ECG en los pacientes, pero sí que es cierto que normalmente estos aparatos se encuentran en las unidades y solamente son utilizados por el personal de manera programada, siendo necesario en casos urgentes acudir a la unidad y trasportarlos hasta el domicilio del paciente para realizar el ECG.

En nuestro caso, hemos utilizado el smartwatch del propio personal de la unidad para realizar ECG en los pacientes en los que por auscultación apreciábamos un ritmo irregular, lo que nos ha permitido detectar episodios de fibrilación auricular; de manera rápida y fiable. Como ejemplo mostramos el trazado realizado con un smartwatch "Apple series 4 con watchOS 6.2.5", que se realizó en un paciente tras auscultar un ritmo cardiaco irregular y que confirma la presencia de una fibrilación auricular no conocida (Figura 1). 
Figura 1: ECG realizado con smartwatch en el que se aprecia fibrilación auricular.

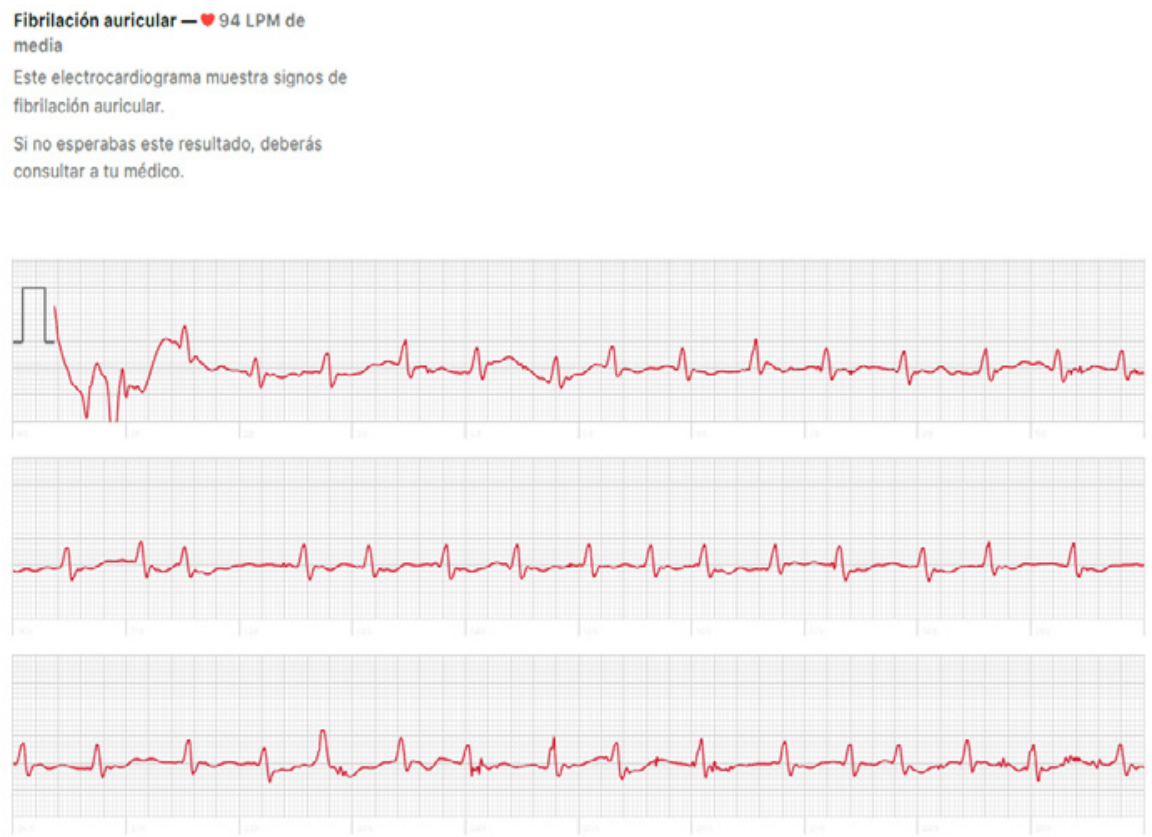

$25 \mathrm{~mm} / \mathrm{s}, 10 \mathrm{~mm} / \mathrm{mV}$, Derivación I, $511 \mathrm{~Hz}$, iOS 13.5, watchOS 6.2 .5 , Watch4,3 - La onda es parecida a la de un ECG de derivacion L. Para obtener mas informacion, consulta las Instrucciones de uso.

Nos parece que estos dispositivos pueden ser de gran utilidad en unidades de hospitalización domiciliaria, ya que permiten realizar una tira de ritmo e incluso como describen en algunos trabajos un ECG de 6 derivaciones con lo que podemos diagnosticar fundamentalmente trastornos del ritmo cardiaco (5), pero también otras alteraciones electrocardiográficas como el alargamiento del segmento QT o incluso elevaciones del segmento ST en el curso de infartos agudos de miocardio. Evidentemente, queda mucho por hacer en este sentido, pero aunque no podamos considerar que estos dispositivos puedan realizar un diagnóstico fiable en todos los casos, sí que permiten realizar una primera aproximación diagnóstica, que puede ser especialmente útil en unidades como las nuestras en las que muchas veces prima la inmediatez y la posibilidad de realizar una técnica diagnóstica sin tener que volver al hospital a por un electrocardiógrafo.

\section{BIBLIOGRAFÍA}

1. Patel NJ, Deshmukh A, Pant S, Singh V, Patel N, Arora S, et al. Contemporary trends of hospitalization for atrial fibrillation in the United States, 2000 through 2010: implications for healthcare planning. Circulation. 2014;129(23):2371-9. DOI: 10.1161/CIRCULATIONAHA.114.008201 
2. Perez MV, Mahaffey KW, Hedlin H, Rumsfeld JS, Garcia A, Ferris T, et al. Large-Scale Assessment of a Smartwatch to Identify Atrial Fibrillation. N Engl J Med. 2019;381(20):1909-17. DOI: 10.1056/NEJMoa1901183

3. Samol A, Bischof K, Luani B, Pascut D, Wiemer M, Kaese S. Recording of Bipolar Multichannel ECGs by a Smartwatch: Modern ECG Diagnostic 100 Years after Einthoven. Sensors (Basel). 2019;19(13):2894. DOI: 10.3390/s19132894

4. Strik M, Caillol T, Ramirez FD, Abu-Alrub S, Marchand H, Welte N, et al. Validating QT-Interval Measurement Using the Apple Watch ECG to Enable Remote Monitoring During the COVID-19 Pandemic. Circulation. 2020;142(4):416-8. DOI: 10.1161/CIRCULATIONAHA.120.048253

5. Marsili IA, Biasiolli L, Masè M, Adami A, Andrighetti AO, Ravelli F, et al. Implementation and validation of real-time algorithms for atrial fibrillation detection on a wearable ECG device. Comput Biol Med. 2020;116:103540. DOI: 10.1016/j.compbiomed.2019.103540 